\title{
Research on Attitude Control and Adjustment of Underwater Platforms Combined with Fuzzy PID
}

\author{
Qing $\mathrm{Li}^{1, \text { a }}$, Yubo Zhang ${ }^{2, \mathrm{~b}}$, Rui Yang ${ }^{3, \mathrm{c}}$ and Wei Wang ${ }^{4, \mathrm{~d}}$ \\ ${ }^{1}$ STRC Dept, China Waterborne Transportation Institute, Beijing, 100088, China; \\ ${ }^{2}$ China Waterborne Transportation Institute, Beijing, 100088, China; \\ ${ }^{3}$ Key Laboratory of Port Equipment \& Control Engineering, Beijing, 100088, China; \\ ${ }^{4}$ Research Institute of Highway Ministry of Transport, Beijing, 100088, China.

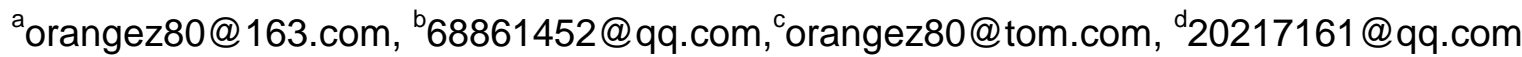 \\ ${ }^{*}$ Corresponding author
}

Keywords: Fuzzy PID, attitude control, attitude adjustment, platforms.

\begin{abstract}
On the basis of the research for attitude control and adjustment, this paper focuses on analyzing and discussing direction control and depth control of underwater platform, builds the course and depth operation models, and receives implementation scheme for attitude control and adjustment of underwater platform by simulation research combined with fuzzy PID control theory.
\end{abstract}

\section{Introduction}

Each year, there are hundreds of sunken ships in the world and submarine marine accidents and aircrafts crashed into the sea occur from time to time. The sea route, especially the busy and narrow navigation channel, may be blocked by sunken ships or objects, so ships cannot enter and leave the port and the port production may be paralyzed in more serious cases. Fuel oil and petroleum products or toxic goods in the cabin of the sunken ships may spill from the body and cause water pollution.

The underwater platform (underwater robot) is an automatic control assembly, it makes three dimensional motions under the water on power provided by cables or propeller mounted on its energy source drive and equipment for lighting and image taking, sonar and other observation equipment, various sensors and professional tools for underwater works can be mounted on it.

\section{Control Model of Underwater Platform}

Analyze and process the experimental data and build the course (direction) kinematic model and depth (depth) kinematic model of underwater platform by MATLAB system identification toolbox.

The transfer function of course simple model of underwater platform is:

$$
G_{1}(s)=\frac{-0.1519 s+1.9821}{1.0613 s^{2}+0.28 s+1}
$$

The transfer function of depth simple model of underwater platform is:

$$
G_{2}(s)=\frac{-0.05 s+0.9}{1.2167 s^{2}+0.4939 s+1}
$$

\section{Impact Parameters on PID Control}

The tuning method for PID control parameters has been basically created after massive experiments and application. Based on the current deviation and deviation change rate of system, on-line tuning of PID control parameters $K_{P}, \frac{K_{P}}{T_{I}}$ and $K_{P} T_{D}$ can be realized by fuzzy adaptive PID controller with fuzzy inference. 
The typical response curve in Figure 1 will be taken as an example to analyze the relationship between deviation, deviation change rate and the three parameters $K_{P}, \frac{K_{P}}{T_{I}}$ and $K_{P} T_{D}$ in each phase of the response curve in order to gain good control performance, thus a fuzzy control rule table is created.

In section OA, the $\mu(t)$ is taken larger in the early phrase of OA in order to accelerate the response speed, while in the position near point $\mathrm{A}, \mu(t)$ shall be smaller to avoid overshoot. That is to say $\mu(t)$ in section $\mathrm{OA}$ is from larger to smaller. In section $\mathrm{AB}$, the $\mu(t)$ direction is opposite to the direction when it is in section OA and the value of $\mu(t)$ increases gradually to prevent the output continuing to deviate from its expected value. ${ }^{\mu(t)}$ shall decrease gradually in section BC to avoid lower overshoot of CD. In section CD, ${ }^{\mu(t)}$ shall

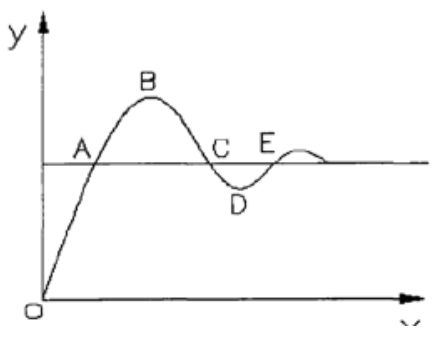

Figure 1 Typical Response Curve keep a smaller value to stabilize the output as soon as possible. In section DE, ${ }^{\mu(t)}$ decreases gradually and the system will tend to be stable.

By analyzing the $\mu(t)$ in each section, we can find the relationship among the PID control parameters $K_{P}, \frac{K_{P}}{T_{I}}$ and $K_{P} T_{D}$ in each phrase, the deviation $e(t)$ and the deviation change rate $d e(t)$. The differentiation element of PID controller can only affect the change rate of system deviation, though the introduced differentiation element can improve the dynamic performance of system, it may also make the system very sensitive to disturbance. Differential action is not taken into consideration here.

Section OA $\left(e(t)_{>0,} d e(t)<0\right)$ : in the early phrase, the speed of the system output trending to the expected value, the faster the better, that is to say it's better to remove the deviation as soon as possible. So $K_{P}$ and $\frac{K_{P}}{T_{I}}$ are taken large. While in the position near point A, $K_{P}$ shall decrease and $\frac{K_{P}}{T_{I}}$ shall be taken an appropriate value at the same time to remove the steady-state deviation.

Section $\mathrm{AB}\left(e(t)_{<0,} d e(t)>0\right)$ : the system output value has exceeded the expected value and the deviation becomes larger. In this section, the overshoot shall be reduced by control action as lower as possible. The integral action is related with the accumulation of deviation, while the deviation $e(t)$ direction in section $\mathrm{AB}$ is opposite to section $\mathrm{OA}$, in this case, the deviation accumulated value is useless and a integral overshoot will be created, so the useless information shall be removed when the deviation sign is changed to clear the accumulated value of integral. Therefore, ${ }^{K_{P}}$ shall be taken larger to make the system output value return to a stable value.

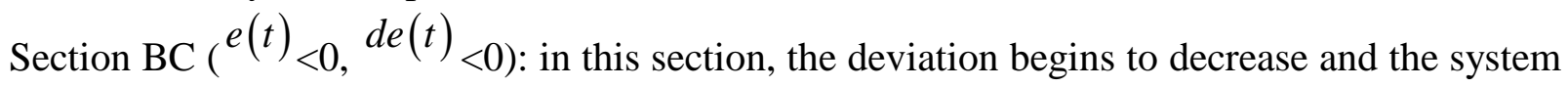
trends to steady-state change by control action. $K_{P}$ and $\frac{K_{P}}{T_{I}}$ shall be decreased appropriately to avoid lower overshoot in section DC. 
Section $\mathrm{CD}\left({ }^{e(t)_{>0},} d e(t)_{<0)}\right.$ : The system have overshoot problem, so $\frac{K_{P}}{T_{I}}$ shall keep smaller and $K_{P}$ shall be increased appropriately to make the system return to the expected value and avoid oscillation.

Section DE $\left(e(t)_{>0} d e(t)>0\right)$ : The system trends to be stable. $K_{P}$ shall keep larger and $\frac{K_{P}}{T_{I}}$ shall keep smaller.

\section{Fuzzy Rule for PID Parameters}

Based on the above analysis, an analytical expression can be used to summarize the relationship between each PID parameter and system deviation and deviation change rate, where an adjustment factor $\alpha$ represents different weighted degrees of PID parameters to system deviation and deviation change rate.

Take the discourse domain of $K_{P}$ and its corresponding deviation and deviation change rate and take the discourse domain of $\frac{K_{P}}{T_{I}}, K_{P} T_{D}$ and their corresponding deviation and deviation change rate. Select each value in the discourse domain of the deviation and deviation change rate, based on the analysis on the relationship between system deviation, deviation change rate and the three parameters $K_{P}, \frac{K_{P}}{T_{I}}$ and $K_{P} T_{D}$, the value of $K_{P}$ can be obtained by selecting different adjustment factors $\alpha$ in different phrases of the system and thus we can get a fuzzy control rule table of parameter $K_{P}$, in the same way, we can get the fuzzy control rule tables of parameters $\frac{K_{P}}{T_{I}}$ and $K_{P} T_{D}$.

This paper built the course and depth fuzzy PID control simulation models of underwater platform with Simulink, as shown in Figure 2 and Figure 3.

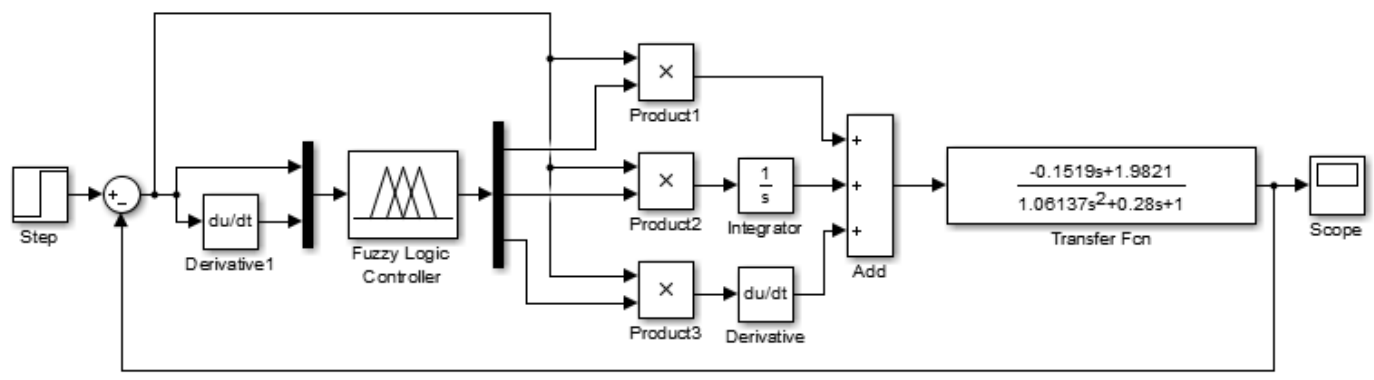

Figure 2 Course Fuzzy PID Control Model

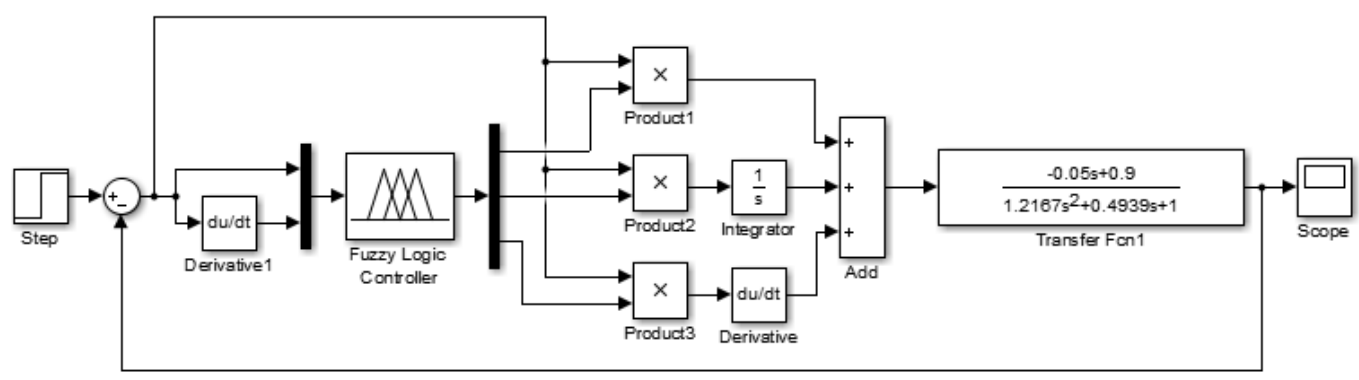

Figure 3 Depth Fuzzy PID Control Model

The most commonly used triangulation rule is selected as the fuzzy rule of course and depth control PID parameters and the control surface diagrams of the three parameters are shown in Figure 4 and Figure 5. 

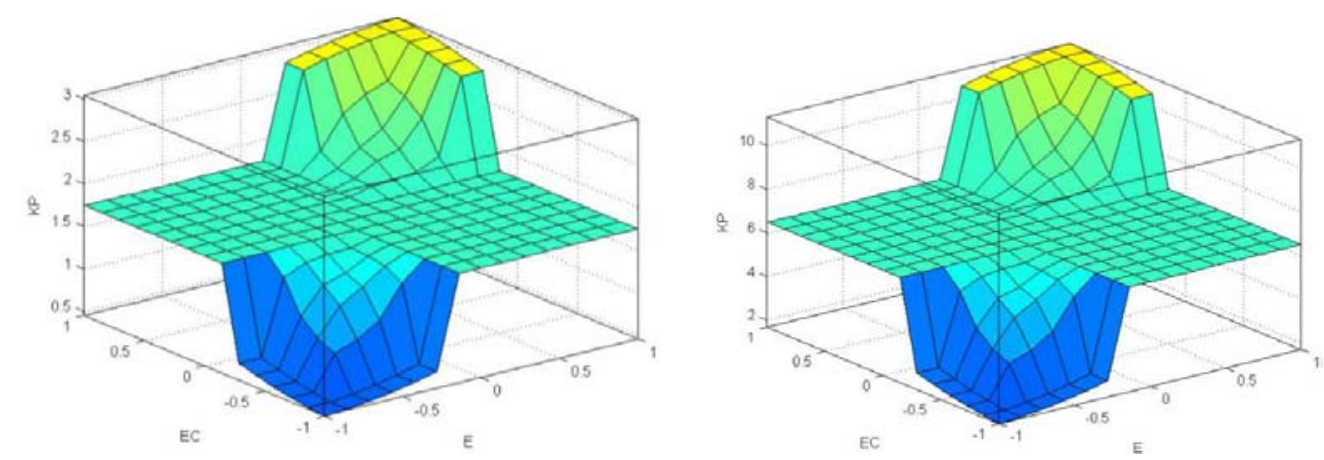

Figure 4 Surface Diagram of Course Control Paramete

Figure5 Surface Diagram of Depth Control Parameter

Step response is also used to evaluate the performance of course and depth fuzzy PID control. The step response curves of course and depth fuzzy PID control are shown in Figure 6 and Figure 7.
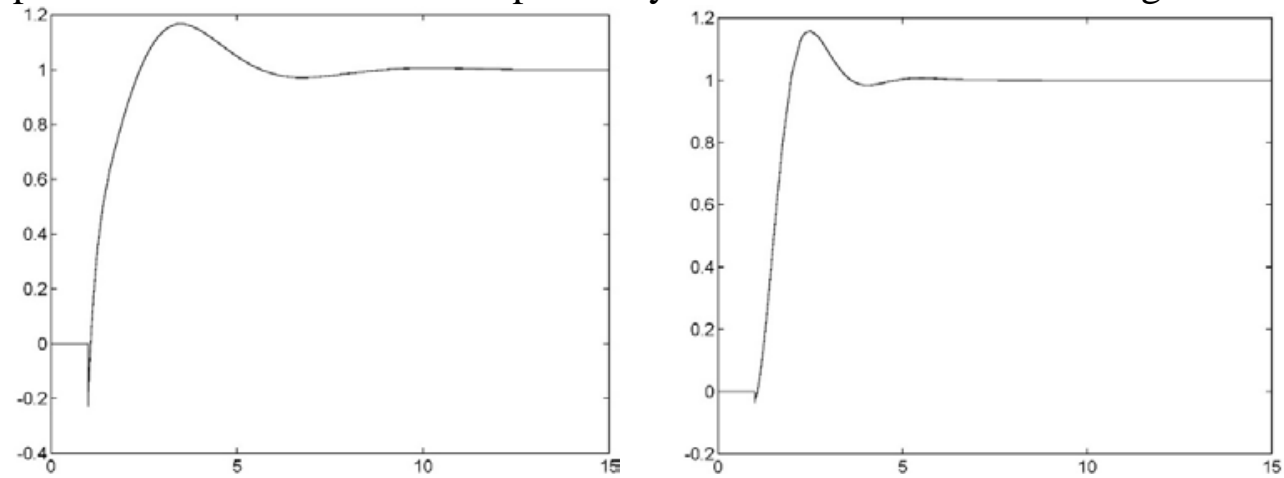

Figure 6 Unit Step Response Curve of Course Fuzzy PID Control

Figure 7 Unit Step Response Curve of Depth Fuzzy PID Control

As seen in Figure 6 and Figure 7, with fuzzy PID algorithm, the course and depth can be controlled quickly and accurately, the rise time, peak time and adjustment time of system step response are very short, overshoot is small, the system steady-state error trends to zero rapidly and the system comes to steady state immediately.

Comparing the course and depth unit step response curves before and after adopting fuzzy PID control algorithm, an obvious result of fuzzy PID control algorithm has been achieved. As long as an estimation model of system and the range of PID parameter are given, we can realize the close-cycle control of the course and depth of underwater platform by automatic matching PID parameter with fuzzy adaptive algorithm.

\section{Summary}

The practical application of fuzzy PID algorithm in attitude control and adjustment of underwater platform is discussed with experimental data and simulation study after repeated underwater experiments and data tests, thus full combination between Theoretical research and experimental research is realized.

\section{References}

[1] JMF Calado, JMGSD Costa, M Bartys. FDI approach to the DAMADICS benchmark problem based on qualitative reasoning coupled with fuzzy neural networks[J]. Control Engineering Practice. 2006

[2] Q Shen, R Leitch. Fuzzy qualitative simulation[J]. IEEE Transactions on Systems Man \& Cybernetics. 1993

[3] W Zhang, H Wang, X Bian, Z Yan, G Xia. The application of self-tuning fuzzy PID control method to recovering AUV[J]. Oceans, 2012:1-5 